\title{
PERCEPCIÓN DE LA LABOR POLICIAL EN LAS CASAS DE JUSTICIA Y LOS CENTROS DE CONVIVENCIA CIUDADANA
}

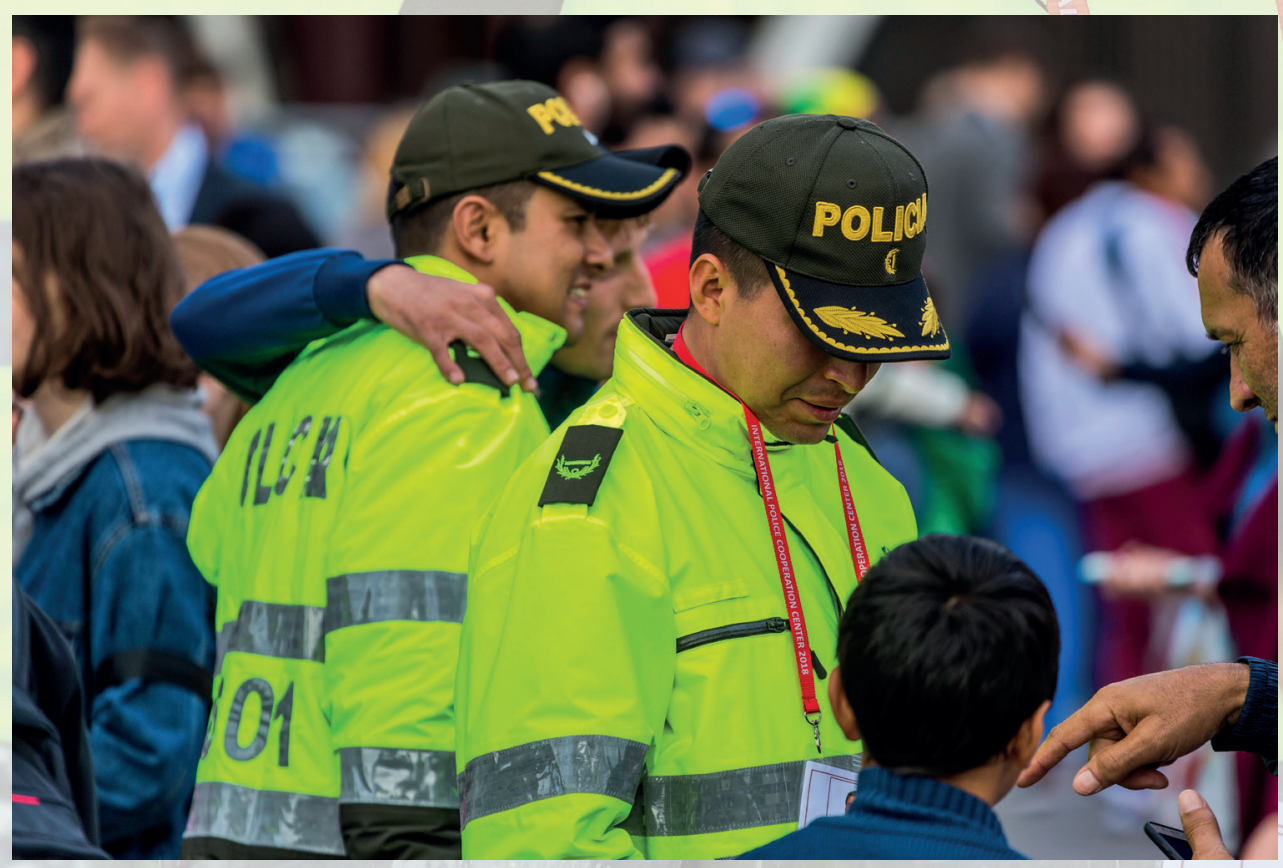





\title{
PERCEPCIÓN DE LA LABOR POLICIAL EN LAS CASAS DE JUSTICIA Y LOS CENTROS DE CONVIVENCIA CIUDADANA*
}

\author{
José Ferney Trujillo Poliandra \\ Claudia Patricia Cáceres Cáceres \\ Centro de Pensamiento Estratégico y Proyección Institucional, \\ Oficina de Planeación, Policía Nacional de Colombia
}

\section{Resumen}

Con la investigación se busca analizar la percepción de la participación de la Policía Nacional de Colombia en el Programa nacional casas de justicia y centros de convivencia ciudadana. Mediante un análisis descriptivo apoyado en entrevistas y encuestas, se examina el alcance de la gestión policial en estos escenarios. Se encontró que, en el marco del programa, hay condiciones favorables para la articulación eficiente con las demás entidades del Estado y los organismos de la sociedad civil en torno a actividades de prevención del delito, solución alternativa de conflictos y promoción de la convivencia ciudadana. Se concluye que la creación de una política institucional tendiente a incrementar y mejorar dicha participación contribuirá a estrechar los vínculos de la Policía Nacional con los ciudadanos y al cumplimiento de su misión constitucional.

Palabras clave: casas de justicia, centros de convivencia ciudadana, manuales de convivencia, mediación policial, métodos alternativos de solución de conflictos.

Los autores: José Ferney Trujillo Poliandra, especialista en gestión territorial de la seguridad de la Escuela de postgrados de la Policía Nacional Miguel Antonio lleras Pizarro. Especialista en Servicio de Policía de Escuela de cadetes de Policía General Francisco de Paula Santander. Politólogo de la Universidad del Cauca. Oficial de la Policía Nacional de Colombia e Investigador del Centro de Pensamiento Estratégico y Proyección Institucional de la Oficina de Planeación de la Policía Nacional.

Claudia Patricia Cáceres Cáceres, doctora en Derecho Canónico. Magister en Ciencias Políticas. Especialista en Derecho Sustantivo y Contencioso Constitucional. Profesional en Derecho y Ciencias socioeconómicas. Investigadora del Centro de Pensamiento Estratégico y Proyección Institucional de la Oficina de Planeación de la Policía Nacional años 2018 y 2019.

Recibido: 3 de marzo 2020; Evaluado: 25 de marzo 2020; Aceptado: 10 de abril 2020

Este artículo es el resultado del proyecto de investigación titulado "Impacto social de la labor de los policías en las casas de justicia y centros de convivencia, en el marco del proceso de modernización y transformación institucional". La entidad financiadora fue la Oficina de Planeación de la Policía Nacional de Colombia. Su fue el 10 de diciembre de 2019. 


\title{
THE PERCEPTION OF POLICE WORK IN HOUSES OF JUSTICE AND COMMUNITY CENTERS
}

\author{
José Ferney Trujillo Poliandra \\ Claudia Patricia Cáceres Cáceres \\ Centro de Pensamiento Estratégico y Proyección Institucional, \\ Oficina de Planeación, Policía Nacional de Colombia
}

\begin{abstract}
This research seeks to analyze the perception of the participation of the Colombian National Police in the national program of Houses of Justice and Civic Coexistence Centers. The article uses a descriptive analysis, interviews, and surveys to analyze the scope of police management in these scenarios. The research found that the framework of the program has favorable conditions for efficient articulation with other State entities and civil society organizations regarding crime prevention activities, alternative conflict resolution, and the promotion of citizen coexistence. It concludes that the creation of an institutional policy to increase and improve this participation would contribute to strengthening the ties of the National Police with citizens and to fulfilling its constitutional mandate.
\end{abstract}

Keywords: houses of justice, civic coexistence centers, coexistence handbooks, police mediation, alternative methods of conflict resolution.

Authors: José Ferney Trujillo Poliandra, specialist in territorial management of the security of the Postgraduate School of the National Police Miguel Antonio Lleras Pizarro. Specialist in Police Service of the General Francisco de Paula Santander Police Cadet School. Political scientist from Universidad del Cauca. Officer of the National Police of Colombia and researcher of the Center for Strategic Thinking and Institutional Projection of the Planning Office of the National Police.

Claudia Patricia Cáceres Cáceres, PhD in Canon Law. Master's in political science. Specialist in Substantive Law and Constitutional Litigation. Bachelor of Law and Socio-Economic Sciences. Researcher at the Center for Strategic Thinking and Institutional Projection of the National Police Planning Office for the years 2018 and 2019.

Received: March 3, 2020; Reviewed: March 25, 2020; Accepted: April 10, 2020 


\title{
PERCEPÇÃO DO TRABALHO POLICIAL NAS CASAS DE JUSTIÇA E NOS CENTROS DE CONVIVÊNCIA CIDADÃ
}

\author{
José Ferney Trujillo Poliandra \\ Claudia Patricia Cáceres Cáceres \\ Centro de Pensamiento Estratégico y Proyección Institucional, \\ Oficina de Planeación, Policía Nacional de Colombia
}

\begin{abstract}
Resumo
Nesta pesquisa, pretende-se analisar a percepção da participação da Polícia Nacional da Colômbia no Programa Nacional Casas de Justiça e Centros de Convivência Cidadã. Mediante análise descritiva apoiada em entrevistas e questionários, o alcance da gestão policial nesses cenários é examinado. Foi verificado que, no âmbito do programa, há condições favoráveis para a articulação eficiente com as demais entidades do Estado e com as organizações da sociedade civil em torno das atividades de prevenção do delito, solução alternativa de conflitos e promoção da convivência cidadã. Conclui-se que a criação de uma política institucional que tende a aumentar e melhorar essa participação contribuirá para estreitar os vínculos da Polícia Nacional com os cidadãos e com o cumprimento de sua missão constitucional.
\end{abstract}

Palavras-chave: Casas de Justiça, Centros de Convivência Cidadã, manuais de convivência, mediação policial, métodos alternativos para a solução de conflitos.

Os autores: José Ferney Trujillo Poliandra, especialista em gestão de segurança territorial pela Escola de PósGraduação Miguel Antonio lleras Pizarro da Polícia Nacional. Especialista em Serviço de Polícia pela Escola de Cadetes de Polícia do General Francisco de Paula Santander. Cientista político da Universidad del Cauca. Oficial da Polícia Nacional da Colômbia e pesquisador do Centro de Pensamento Estratégico e Projeção Institucional do Escritório de Planejamento da Polícia Nacional.

Claudia Patricia Cáceres Cáceres, doutora em Direito Canônico. Mestre em Ciência Política. Especialista em Direito Substantivo e Contencioso Constitucional. Advogada e profissional em Ciências Socioeconômicas. Pesquisadora do Centro de Pensamento Estratégico e Projeção Institucional do Escritório de Planejamento da Polícia Nacional para os anos de 2018 e 2019.

Recebido: 3 de março 2020; avaliado: 25 de março 2020; aceito: 10 de abril 2020 


\section{Introducción}

El Decreto 1477 de 2000 crea las casas de justicia y los centros de convivencia ciudadana, dentro de los cuales la Policía Nacional tiene un rol estratégico, con el objetivo de promover y garantizar la convivencia ciudadana en el país. En la actualidad, no existe un diagnóstico acerca de la participación de la Policía Nacional de Colombia en dichos espacios de cara a fortalecer la promoción de la convivencia ciudadana y de robustecer la debida aplicación del Código Nacional de Seguridad y Convivencia Ciudadana, como un mecanismo para tramitar los conflictos sociales y, por ende, facilitar el acceso a la justica para los ciudadanos.

Para este análisis se elaboró un estudio descriptivo, basado en la recolección de información que permitiera formular una estrategia de gestión para los funcionarios de la Policía Nacional en relación con las casas de justicia y los centros de convivencia ciudadana. Como instrumentos específicos de investigación se implementaron dos encuestas: una dirigida a los comandantes de la Policía Nacional y otra, a los coordinadores de casas de justicia y centros de convivencia ciudadana, cuya gestión es orientada desde la Dirección de métodos alternativos de solución de conflictos, instancia encargada del programa.

Este proceso se complementó con entrevistas a expertos acerca de la operatividad, la sostenibilidad y la conveniencia, con el fin de proponer una estrategia de gestión que posibilite el mejor aprovechamiento de las casas de justicia y de los centros de convivencia ciudadana por parte de la institución policial.

El artículo presenta, como punto de partida, la perspectiva de la convivencia ciudadana como eje de la prestación del servicio de policía, piedra angular de las acciones institucionales de la Policía Nacional; luego se expone la alineación de las acciones frente a la convivencia ciudadana con el Plan Nacional de Desarrollo 2018-2022, para adentrarse en el análisis del Programa nacional de casas de justicia y centros de convivencia ciudadana del Ministerio de Justicia y del Derecho. Por último se abordarán los resultados del estudio adelantado en estos espacios institucionales, así como los aportes y las recomendaciones para el mejoramiento y la articulación con la Policía Nacional de Colombia. 


\section{Convivencia ciudadana: eje de la prestación del servicio de policía}

La convivencia ciudadana, en términos generales, es la construcción de relaciones cotidianas que permiten el desarrollo de una comunidad en donde los intereses individuales y colectivos logren armonizarse para construir una sociedad con un tejido fuerte y corresponsable. Páramo puntualiza:

Por lo general la convivencia se entiende como la interacción armoniosa, respetuosa, amable, social, entre las personas y con los animales en un mismo ambiente, el barrio o la ciudad, en donde se comparten reglas de urbanidad en el marco muchas veces de una normativa. Además se hace referencia a la convivencia como el respeto al otro. Entender que se tienen derechos pero también deberes para con los demás. Compartir el espacio físico para vivir bien y que el otro también pueda hacerlo. Aceptar al otro que es diferente a mí, pero tiene el mismo derecho que yo a ser diferente. ${ }^{1}$

Sin embargo, esta perspectiva tiende a fragmentarse dentro las dinámicas naturales de las relaciones humanas. Entonces, la estructuración de cuerpos normativos orienta cuáles son los comportamientos aceptados para la vida en comunidad y es la Policía la institución que, por su razón de ser, se ocupa de su cumplimiento. Bello Montes sostiene:

[...] el desarrollo de las cuatro categorías que la conforman — seguridad, tranquilidad, moralidad, ecología — es el objeto central de los posibles escenarios que deberá enfrentar el país en las próximas décadas, y por consiguiente el cuerpo policial, para garantizar el ejercicio de los derechos y deberes ciudadanos, al igual que el mantenimiento de la paz y la convivencia de la ciudadanía. ${ }^{2}$

Expresa que la convivencia ciudadana implica el desarrollo de un orden jurídico y político configurado a partir de los deberes y los derechos de los ciudadanos. Para Suárez, la convivencia "se vincula de manera activa con la necesidad de generar cultura ciudadana o la apropiación de comportamientos sociales que faciliten el cumplimiento de acuerdos, la obediencia a la norma, la confianza en las instituciones

Pablo Páramo y Andrea Burbano, Convivencia ciudadana en ciudades latinoamericanas (Bogotá: Universidad Pedagógica Nacional, Universidad de la República Uruguay, 2019), 19.

2 Catalina Bello, "Desafíos y estado futuro de la convivencia en Colombia al 2025", Criminalidad 56, núm. 2 (2014): 331, https://dialnet.unirioja.es/servlet/articulo?codigo=5125433 (acceso agosto 15, 2019). 
y la vida en sociedad". ${ }^{3}$ La cultura ciudadana, a su vez, tiene que ver con valores como la "actitud hacia la ley, los acuerdos, la solidaridad, la confianza interpersonal y en las instituciones, la organización social y la seguridad ciudadana". . Otra acepción traída a colación por la investigadora hace referencia a la convivencia democrática, la cual tiene que ver con las relaciones de poder entre los ciudadanos y el Estado y con la existencia de normas que garanticen los derechos ciudadanos dentro de un Estado social de derecho.

Dicho lo anterior, las estrategias para promover la convivencia serían, entre otras, las siguientes: aumentar la actitud positiva de la ciudadanía respecto a la ley y a las normas de convivencia; mejorar su cumplimiento; incrementar los índices de seguridad ciudadana, estimular la conciencia de los factores de riesgo y la cooperación con las autoridades y mejorar las actitudes y los comportamientos de la ciudadanía con respecto a la concertación y al cumplimiento de acuerdos; mejorar los grados de tolerancia y solidaridad; aumentar la organización social y la participación de la ciudadanía, e incrementar la confianza interpersonal y en las instituciones públicas. Finalmente, es imperativo fortalecer los canales de coordinación entre autoridades territoriales y policiales, para garantizar el desarrollo de políticas públicas de seguridad y convivencia acordes con las necesidades de los municipios. ${ }^{5}$

Para robustecer las acciones de convivencia, con el Decreto 1477 de 2000 se estableció el Programa nacional de casas de justicia y convivencia ciudadana. Los dos elementos constitutivos del Programa nacieron con visiones similares: el primero, con un enfoque de justicia, y el segundo, de seguridad ciudadana, pero las casas de justicia han contado con mayor voluntad política para su desarrollo e implementación, por lo cual hoy pueden mostrar avances palpables.

La gestión de la Policía Nacional en casas de justicia está circunscrita a prestar apoyo a las autoridades, en especial para atender las solicitudes emitidas por los inspectores de policía o para hacer intervenciones relacionadas con protección de mujeres, niños, niñas y adolescentes. En este ámbito se destaca la mediación policial, un mecanismo que ha sido diseñado con el objetivo de resolver conflictos ligados a la convivencia diaria, evitar procesos penales o administrativos dispendiosos y facilitar el acceso a la justicia.

Jairo Suárez, Avances de la ciencia de policía en América Latina (Bogotá: Policía Nacional de Colombia, 2009), citado en Bello, "Desafíos y estado futuro de la convivencia", 322-323.

4 Bello, "Desafíos y estado futuro de la convivencia", 325.

5 Bello, "Desafíos y estado futuro de la convivencia", 327. 
Frente a los problemas que tienen los ciudadanos para acceder a la justicia, Camacho ha identificado una serie de retos que deben ser superados. Entre ellos destaca: los mecanismos de solución alternativa de conflictos son lejanos al ciudadano; el diseño institucional del sistema de administración de justicia dificulta la articulación; la ausencia del Estado en zonas marginales; el presupuesto limitado; la indiferencia, la corrupción y la incapacidad institucional; la debilidad en la formación para la solución de conflictos; la poca sensibilidad de los funcionarios ante la importancia social de su gestión; la desconfianza en las instituciones; la discriminación; el desconocimiento de la ley, y los costos relacionados con el acceso a la justicia aunados a la pobreza. Estas son algunas de las barreras que aún no han podido ser superadas; sin embargo, se han desarrollado estrategias para reducirlas por medio de la implementación del Plan Sectorial de la Rama Judicial 2011-2014. ${ }^{6}$

Las casas de justicia son instrumentos idóneos para superar estas barreras y la labor de la Policía Nacional en estos espacios es una gran oportunidad para contribuir al acceso a la justicia, en especial porque cuenta con funcionaros competentes y con capacidades asertivas en materia de mediación policial.

La Policía Nacional, en el marco del Programa, considera que el diagnóstico de conflictividad puede convertirse en una herramienta fundamental para acercarse a la comunidad y para participar en el diseño de los planes y programas de seguridad de cada municipio, entre ellos, los relacionados con los espacios institucionales que aquí se analizan. De forma similar, se aborda el estudio desarrollado por Ahumada y Manzano, desarrollado en la provincia de La Rioja por medio de una encuesta a pobladores, a operadores de justicia del municipio y a funcionarios de otras instancias estatales respecto al acceso a la justicia. ${ }^{7}$

Los resultados obtenidos demuestran que un alto porcentaje de ciudadanos prefiere no hacer nada frente al conflicto, si bien ahora tiene mayor acceso a la información acerca de sus derechos y de la manera como protegerlos; sin embargo, muchos ciudadanos encontraron que los trámites eran engorrosos y que el acceso a la justicia era lejano, por lo cual prefirieron no resolverlo.

6 Lina Camacho, "Acceso a la justicia en Colombia. Condiciones de posibilidad y criterios de gestión" (Tesis de maestría inédita, Universidad Nacional de Colombia, 2013).

7 Carolina Ahumada y Alejandra Manzano, Estudio exploratorio sobre acceso a la justicia en la localidad de Sanagasta - Provincia de La Rioja (Buenos Aires: Instituto de Estudios Comparados en Ciencias Penales y Sociales, 2016). 


\section{Alineación con el Plan Nacional de Desarrollo 2018-2022. Pacto por Colombia, pacto por la equidad}

En el Plan Nacional de Desarrollo 2018-2022 se privilegian los sectores de seguridad y de justicia; en su primer punto establece el "Pacto por la legalidad: seguridad efectiva y justicia transparente para que todos vivamos con libertad y en democracia", en el cual precisa que la legalidad se basa en dos pilares: la seguridad y la justicia. Se requiere, entonces, una justicia "eficaz, eficiente y efectiva". 8

El Plan Nacional de Desarrollo, como faro orientador de la política pública, propone acciones prioritarias en cuanto a la seguridad y la justicia, entre las que se cuentan la revisión y promulgación del Código nacional de seguridad y convivencia ciudadana, centrado en los mecanismos para disminuir las problemáticas en este ámbito. Se destaca que:

[...] favorece la acción preventiva frente a comportamientos contrarios a la convivencia, se ha presentado (1) una baja apropiación y compromiso por parte de las autoridades locales en la socialización e implementación del código; (2) una falta de apropiación del mismo por parte de la ciudadanía; (3) falencias en los procesos de reglamentación nacional-territorial y de recaudo de las medidas correctivas; y (4) falencias en los procedimientos y procesos a cargo de las autoridades de policía, que han dificultado el trabajo articulado entre la Policía Nacional y los inspectores de policía y corregidores. Esto se ve reflejado en el comportamiento que tuvieron durante el 2018 las conductas contrarias a la convivencia, donde el consumo de bebidas alcohólicas en el espacio público y el porte de armas, de elementos cortopunzantes y semejantes, fueron los comportamientos que más afectaron la convivencia en el país. ${ }^{9}$

En concordancia con la orientación del fortalecimiento de la seguridad y la convivencia ciudadana, entendida como un servicio público que debe prestar el Estado paralelo a la construcción de legalidad, el pacto por la legalidad tiene entre sus objetivos centrales:

Promover estrategias dirigidas a mejorar, fortalecer y garantizar condiciones de convivencia y seguridad ciudadana en los centros urbanos y el campo,

Departamento Nacional de Planeación, Bases del Plan Nacional de Desarrollo 2018-2022. Pacto por Colombia, pacto por la equidad (Bogotá: Autor, 2018), 12.

9 Departamento Nacional de Planeación, Bases del Plan Nacional de Desarrollo, 16. 
para lo cual los ministerios de Defensa y del Interior en coordinación con las entidades pertinentes, formularán la Política Nacional de Convivencia y Seguridad Ciudadana. ${ }^{10}$

Esta orientación de la política pública se cristaliza territorialmente con la articulación de los procesos de asistencia y asesoría en el marco de las estrategias de planeación territorial de seguridad y convivencia ciudadana, con los procesos de seguimiento y evaluación de los planes integrales de seguridad y convivencia. ${ }^{11}$ Esto determina un marco normativo que pretende no solamente el fortalecimiento de la política, sino la garantía de transparencia y probidad en la prestación del servicio.

En cuanto al alcance en el ámbito del fortalecimiento institucional en el campo de la seguridad y la convivencia ciudadana, uno de los elementos centrales radica en la reducción de las barreras de acceso a la justicia en los territorios, para garantizar de esta manera soluciones a múltiples necesidades de los ciudadanos a partir de métodos alternativos que disminuyan los procesos dentro de la Rama Judicial:

El Estado debe buscar superar las barreras de acceso a la justicia que se generan cuando las comunidades y empresas perciben la justicia alejada de su cotidianidad. Concebir la oferta desde lo local, teniendo en cuenta particularidades territoriales; y promover e incentivar el uso de métodos de resolución de conflictos que resuelvan necesidades jurídicas de los ciudadanos (sin tener que acudir a la Rama Judicial) permitirán una mejor convivencia y la reconstrucción del tejido social mediante el diálogo y la concertación. ${ }^{12}$

Acerca de las casas de justicia y convivencia ciudadana, se afirma que, a pesar del gran progreso que han significado en materia de acceso a la justicia, persisten dificultades como la ausencia de una caracterización de los municipios, necesaria para diseñar un modelo que responda a los requerimientos locales de acceso a la justicia y de resolución de conflictos, relacionados con las dificultades propias de la ruralidad, como la diseminación de sus habitantes. Otra dificultad es el desconocimiento del impacto de los métodos alternativos de solución de conflictos en materia de reconstrucción del tejido social y mejoramiento de la convivencia, en especial por el bajo número de operadores de estos medios alternativos y la dispersa normatividad sobre la materia.

Departamento Nacional de Planeación, Bases del Plan Nacional, 32.

Departamento Nacional de Planeación, Bases del Plan Nacional, 14.

12 Departamento Nacional de Planeación, Bases del Plan Nacional, 42. 


\section{Programa nacional de casas de justicia y convivencia ciudadana del Ministerio de Justicia y del Derecho}

El 13 de mayo de 2019 fue expedido, por el Ministerio de Justicia y del Derecho de la República de Colombia, el manual denominado Implementación y sostenibilidad del Programa nacional de casas de justicia y convivencia ciudadana, el cual fue elaborado por la Dirección de métodos alternativos de solución de conflictos, a cuyo cargo se encuentra el Programa. El manual abarca aspectos fundamentales para la implementación, como los objetivos, la misión, la visión, los principios, el marco normativo, los requisitos para su implementación y la guía para su sostenibilidad, entre otros.

En primer lugar, el objetivo central de las casas de justicia y convivencia ciudadana es acercar la justicia a la ciudadanía para promover y garantizar el acceso a la misma, sin restricciones, de forma gratuita y con las facilidades suficientes. Por su parte, los centros de convivencia ciudadana son definidos como un:

Modelo de atención (infraestructura y equipamiento) donde se promueve la convivencia ciudadana a través de la articulación de entidades prioritariamente de orden local y de la justicia comunitaria, que desarrollan programas psicosociales y de acceso a la justicia a través de mecanismos formales y alternativos relacionados con la prevención, la atención y la resolución de conflictos, con el fin de contribuir a la reconstrucción y sostenibilidad del tejido social en las comunidades, utilizando la lúdica y la lectura. ${ }^{13}$

Estos espacios se acompañan con modelos de gestión creados para facilitar el acceso a la justicia y establecen los lineamientos para la atención de los ciudadanos y para implementar mecanismos de prevención, sobre todo en aquellos lugares de alta conflictividad y vulnerabilidad social. Además de los propósitos mencionados, se pretende desarrollar estrategias de prevención orientadas al fortalecimiento de la convivencia ciudadana y al restablecimiento del tejido social.

13 Jimmy Sánchez et al., Manual para la implementación y sostenibilidad del Programa nacional de casas de justicia y convivencia ciudadana (Bogotá: Ministerio de Justicia y del Derecho, 2019), 4. 
El Programa tiene los siguientes fines:

Ampliar la cobertura en el acceso a la justicia, prioritariamente, para las comunidades en zonas marginales de las cabeceras municipales y en zonas rurales apartadas. Impulsar una cultura de convivencia pacífica y de respeto al derecho ajeno entre los habitantes de las zonas de influencia del programa. Contribuir a la construcción de una convivencia pacífica con la participación de la ciudadanía. Promover el uso y la difusión de los mecanismos alternativos de solución de conflictos. Asesorar y orientar a la comunidad en el uso del servicio público de la justicia, acorde con sus necesidades. Desarrollar programas de prevención en los diferentes tipos de violencias. ${ }^{14}$

En el campo de la implementación, se plantea la necesidad de crear espacios de prestación de servicios de justicia comunitaria y no formal que faciliten el acceso a la comunidad, para ampliar, de esta manera, el acceso a mecanismos alternativos de resolución de conflictos que fomenten la cultura de la convivencia pacífica, la protección de los derechos humanos y el análisis de la conflictividad social. ${ }^{15}$

El Ministerio procura que el Programa tenga la mayor cobertura posible en todo el territorio nacional; por ello, se encarga de los procesos de coordinación, la capacitación necesaria para su implementación y del apoyo en la comunicación y difusión del mismo, de cara a promover la participación ciudadana. Adicionalmente, el Ministerio ha delimitado sus líneas estratégicas: derechos humanos; componente étnico en la política pública de acceso a la justicia; fortalecimiento institucional; prevención y atención de violencia contra niños, niñas y adolescentes; prevención y atención de la violencia contra la mujer y la familia; jornadas móviles de justicia y convivencia ciudadana, y mecanismos alternativos de solución de conflictos.

En cuanto a su fundamento normativo, el Programa obedece al desarrollo del Artículo 229 de la Constitución Política, el cual consagra: "Se garantiza el derecho de toda persona para acceder a la administración de justicia. La ley indicará en qué casos podrá hacerlo sin la representación de abogado", ${ }^{16}$ así como al Artículo 91 de la Ley 136 de 1994, según el cual los alcaldes, además de estar encargados de ejecutar acciones tendientes a garantizar la promoción de la solidaridad y la convivencia entre los habitantes del municipio, deben adelantar acciones para:

Sánchez et al., Manual para la implementación y sostenibilidad, 7.

Sánchez et al., Manual para la implementación y sostenibilidad, 7.

16 República de Colombia, Constitución Política (Bogotá: Legis, 1991), art. 229. 
[...] lograr el mejoramiento de la gestión local, promover la armoniosa concurrencia de la Nación, las entidades territoriales, las autoridades ambientales y las instancias y autoridades administrativas y de planificación en el cumplimiento de las obligaciones constitucionales y legales en materia territorial. En especial, contribuir en el marco de sus competencias, con el fin de garantizar el despliegue de infraestructuras para lograr el desarrollo y la competitividad nacional de conformidad con lo dispuesto en el Plan Nacional de Desarrollo. ${ }^{17}$

Específicamente, el Programa se encuentra regulado en el Decreto 1477 de 2000 como un mecanismo para garantizar el acceso de los ciudadanos a la justicia, que fue incorporado en el Título IV del Decreto 1069 de 2017, único reglamentario del sector justicia, bajo el acápite denominado "Métodos alternativos de solución de conflictos". ${ }^{18}$

Hoy en día operan 104 casas de justicia en 88 municipios del país, donde se resuelven conflictos menores ligados a la convivencia, entre los cuales se pueden mencionar: conflictos familiares, pérdida o ausencia de documentos, problemas penales, conflictos entre vecinos, no pago de arriendo y servicios públicos, problemas civiles, problemas laborales, atención, apoyo y orientación psicológica, solicitud de información, y violación de derechos humanos por parte de miembros de la fuerza pública. ${ }^{19}$

Por su parte, los centros de convivencia ciudadana son de más reciente implementación. En la actualidad existen 33 en operación en el mismo número de municipios, distribuidos en 18 departamentos del país; el primero de ellos fue puesto en marcha en febrero de 2002, en Barrancabermeja. El objetivo general de los centros consiste en "incentivar el rescate de los valores ciudadanos, de cooperación, solidaridad y organización social, como requisito fundamental para el rechazo de la violencia y la consolidación de la gobernabilidad global", ${ }^{20}$ promover la defensa de los ciudadanos frente a conflictividades y violencias, agilizar la respuesta institucional

17 Colombia, Congreso de la República, Ley 136 de 1994, "Por la cual se dictan normas tendientes a modernizar la organización y el funcionamiento de los municipios" (Bogotá: Diario Oficial núm. 41.377, 2 de junio de 1994), art. 91.

18 Colombia, Presidencia de la República, Decreto 1477 de 2000, "Por el cual se adopta el Programa Nacional Casas de Justicia" (Bogotá: Diario Oficial núm. 44.113, 3 de agosto de 2000).

19 Sánchez et al., Manual para la implementación y sostenibilidad, 20.

20 Rodolfo de Jesús Díaz et al., Guía para la implementación del Programa nacional de casas de justicia y centros de convivencia ciudadana (Bogotá: Ministerio de Justicia y del Derecho, 2019), 4. 
del Estado y estimular múltiples formas de consolidación de las relaciones sociales y la convivencia pacífica.

En cuanto al funcionamiento general de dichas instancias, el rol de la Policía Nacional es determinante e insustituible. Su labor consiste en salvaguardar al ciudadano en materia de seguridad y convivencia ciudadana; intervenir directamente en la protección de las víctimas de violencia intrafamiliar en colaboración con la Comisaría y la Defensoría de Familia; tramitar y decidir querellas civiles policivas por conflictos entre particulares en casos de perturbación a la posesión, propiedad o tenencia de bienes muebles o inmuebles; expedir protecciones policivas, y practicar comisiones impartidas por juzgados y fiscalías.

Igualmente, la Policía Nacional de Colombia hace presencia en las casas de justicia por medio de la Policía de Infancia y Adolescencia. Durante 2018, se consolidó la operación de las casas de justicia de Buga, Cajicá, Cartagena, Caucasia, Chía, El Bagre, Frontino, Girardot, Ipiales, La Plata, Mocoa, Supía, Tunja y Paipa; recibió 1.202 solicitudes en todo el país, provenientes de 1.101 personas, de las cuales fueron atendidas 644 .

En el primer semestre de 2019, la Policía Nacional recibió en casas de justicia un total de 414 solicitudes provenientes de 388 personas, de las cuales fueron atendidas 181. Aunque las categorías para la clasificación de la información cambiaron, se sigue presentando el mismo inconveniente que se mencionó, referente a la falta de unidad para la recolección de la información. Los centros de convivencia ciudadana, a diferencia de las casas de justicia, no se encuentran regulados por decreto y, en cuanto a su operatividad, se tienen en cuenta, en general, los mismos criterios que se aplican para las citadas casas. Según la doctora Hilda Rojas, del Ministerio de Justicia y del Derecho, para la puesta en marcha de estos centros se requiere que el respectivo municipio o ente territorial tenga una población entre 30.000 y 91.000 habitantes, mientras que para erigir una casa de justicia se necesita de un mínimo de 129.000 habitantes. Entre otros factores ya explicados someramente, para la implementación de estos espacios, se destaca la capacidad institucional del municipio, de cara a poder ofrecer los servicios de manera sostenible. ${ }^{21}$

En los dos casos, tanto para poner en marcha una casa de justicia, como un centro de convivencia ciudadana, se requiere que dicho proyecto esté contemplado en

21 Hilda Rojas, comunicación personal, 15 de agosto de 2019. 
el Plan de desarrollo local y en el respectivo proyecto de presupuesto. Se resalta que siempre se exigirá la elaboración de un estudio de conflictividad a cargo de la Policía y, por ende, conviene que este elemento se encuentre presupuestado. En este sentido, el análisis de la conflictividad social que se refleja en las estadísticas de comportamientos contrarios a la convivencia registrados en la aplicación del Código Nacional de Seguridad y Convivencia Ciudadana permitirá identificar las conductas con mayor prevalencia, al igual que su volumen de ocurrencia. Este resultado serviría además como insumo al Ministerio de Justicia y del Derecho para evaluar la dimensión de la conflictividad y el alcance del Programa nacional de casas de justicia y centros de convivencia frente a la misma.

\section{Percepción de los comandantes de Policía y de los coordinadores del Ministerio de Justicia y del Derecho acerca de las casas de justicia y los centros de convivencia ciudadana}

\subsection{Encuesta a los comandantes de Policía}

Durante la investigación se encuestaron cuarenta comandantes de Policía y se obtuvo respuesta de veintiún uniformados. Se quiso establecer el grado de importancia de las casas de justicia como mecanismo de prevención de la delincuencia, gracias a un adecuado trámite institucional de la conflictividad social. La consulta arrojó los siguientes resultados:

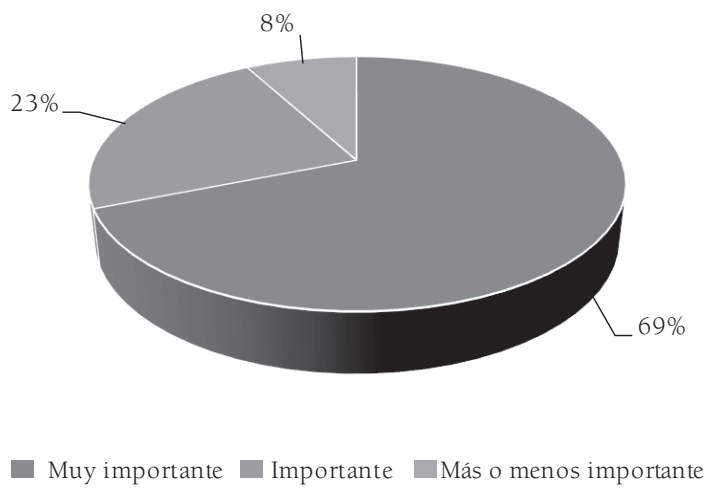

Figura 1. Importancia de las casas de justicia como mecanismo de prevención de la delincuencia.

Fuente: elaboración propia. 
Los datos obtenidos muestran que, para los comandantes consultados, las actividades realizadas en las casas de justicia son vitales en el marco de la reducción de conflictividades En la misma consulta, frente a los centros de convivencia ciudadana se obtuvo la misma tendencia de respuesta. Sin embargo, esta percepción contrasta con el despliegue institucional, específicamente con la cantidad de miembros de la Policía Nacional que son asignados al cumplimiento de labores en las casas de justicia.
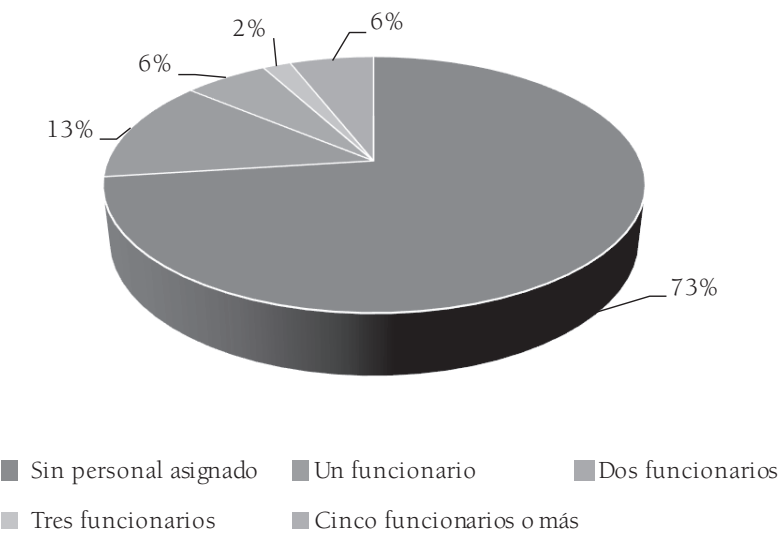

Figura 2. Número de funcionarios asignados a las casas de justicia por parte de los comandantes de Policía.

Fuente: elaboración propia.

Es contradictorio que un escenario con esta relevancia cuente con una baja cantidad de personal de asignación por parte de la Policía Nacional. Es posible inferir que la reducción de los nuevos enlistados a la institución y la priorización a otras necesidades de servicio han disminuido la cantidad de recursos humanos disponible tanto en las casas de justicia como en los centros de convivencia ciudadana; como fue posible constatar en la consulta con los comandantes, solo dos de ellos reportaron haber asignado un uniformado en estos espacios.

En general, los asuntos atendidos por la Policía, en virtud de solicitudes que provienen de las casas de justicia, corresponden, en su orden, a conflictos entre vecinos, violencia intrafamiliar, lesiones personales sin secuelas, inasistencia alimentaria, hurto simple, e injuria y calumnia. Otro servicio prestado por la Institución en estas casas consiste en dictar cursos pedagógicos derivados de comparendos de tránsito. 
En cuanto a las recomendaciones a la Institución recibidas por parte de los comandantes de Policía encuestados se resaltan: coordinar, desde el escenario central con el Ministerio de Justicia y del Derecho, la creación de más casas de justicia y centros de convivencia ciudadana y, a largo plazo, cubrir todo el territorio nacional; dotar a los funcionarios con medios suficientes para su gestión — tecnológicos, transporte y demás insumos necesarios- y brindarles capacitación adecuada; solicitar a las autoridades gubernamentales una mejor articulación con la Policía y la promoción de los servicios de las casas de justicia; aumentar la coordinación interinstitucional para mejorar la convivencia y la seguridad; establecer su funcionamiento las veinticuatro horas del día y los siete días de la semana; garantizar infraestructura adecuada, así como la dotación y la sostenibilidad de las casas de justicia; incluir salas de mediación policial, y aumentar el grado de compromiso por parte de los inspectores de policía.

Respecto a los centros de convivencia ciudadana, señalaron la importancia de asignar funcionarios de Policía permanentes en los espacios; aumentar la capacitación en materia de convivencia; lograr mayor promoción por parte de las Alcaldías locales; crear gestores de la Policía Nacional encargados de apoyar los centros; mejorar las instalaciones y no postergar su implementación y, por último, diseñar planes y programas que involucren a la comunidad e incrementar el número de funcionarios calificados por parte de las instituciones que conforman el centro: psicólogos, trabajadores sociales, etc.

\subsection{Encuesta a los coordinadores de casas de justicia y centros de convivencia ciudadana}

Con la colaboración del Ministerio de Justicia y del Derecho, se diligenció una encuesta entre todos los 137 coordinadores de casas de justicia y centros de convivencia ciudadana y se obtuvo respuesta de 111 funcionarios. Para comenzar, un $55 \%$ de los encuestados afirmó que su casa de justicia o centro de convivencia ciudadana no cuenta con la presencia de la Policía Nacional, mientras que el 45 \% restante aseguró contar con el apoyo de la Institución. Aquellos que reconocen la presencia de la Policía Nacional indicaron que los servicios prestados por los miembros de la Institución se desglosaban en: 


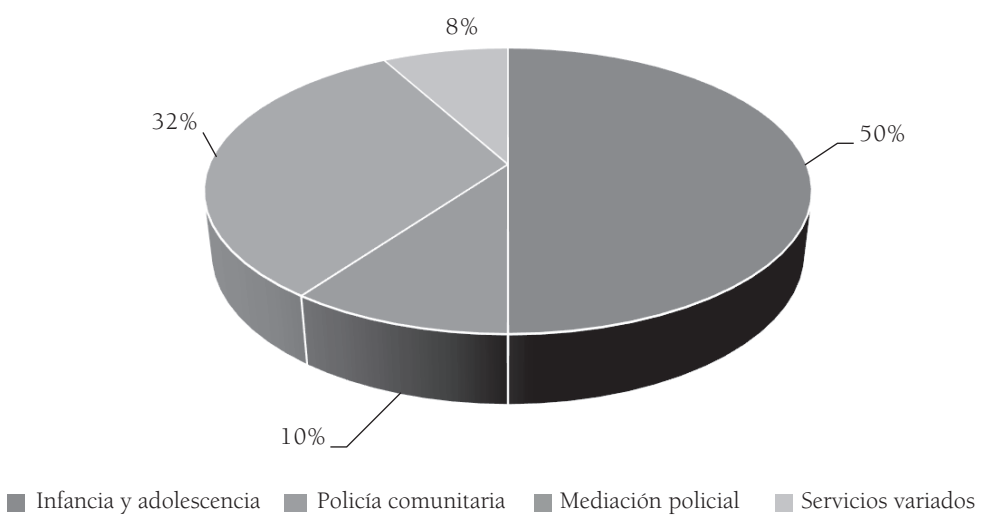

Figura 3. Tipos de servicios prestados por la Policía Nacional de Colombia en las casas de justicia y los centros de convivencia ciudadana

Fuente: elaboración propia.

Se puede destacar que, en el marco de sus actividades, se adelantan acciones como: medidas a protegidos, labores de inteligencia, campañas de prevención de reclutamiento de menores por grupos al margen de la ley, asesorías en materia de aplicación del Código Nacional de Seguridad y Convivencia Ciudadana, educación ciudadana, turismo y ambiente, recepción de denuncias, apoyo al centro de conciliación y despachos comisorios, entre otros. Por último, un 78 \% de los encuestados indicó que la Policía Nacional, en su despliegue, era cercana a los ciudadanos.

Las atenciones prestadas por los policías en estos espacios abarcan: violencia intrafamiliar, lesiones personales sin secuelas, conflictos entre vecinos, hurto simple, desorden en la vía o lugares públicos, inasistencia alimentaria, custodia y cuidado de niños, niñas y adolescentes, contaminación visual y otros.

\section{Hallazgos, comentarios y recomendaciones}

La investigación permitió evidenciar una serie de hallazgos determinantes para el fortalecimiento de la policía nacional en las casas de justicia y los centros de convivencia ciudadana:

1. El formato para el diagnóstico de conflictividad de los municipios que sirve como base para la solicitud de creación de una casa de justicia o centro de convivencia ciudadana data del año 2016; por lo tanto, debería ser actualizado 
con base en la reciente investigación y como desarrollo institucional para la elaboración de planes integrales departamentales y municipales de convivencia y seguridad ciudadana.

2. La labor realizada por los integrantes de la Policía Nacional no es conocida por la comunidad en las casas de justicia y los centros de convivencia ciudadana. Requiere mayor difusión por parte de las instituciones.

3. La Policía Nacional debería promover la creación de las instancias del Programa nacional de casas de justicia y centros de convivencia ciudadana en los municipios, con una actitud proactiva que generaría un efecto positivo en su imagen institucional y en su credibilidad y sería una manera de profundizar sus vínculos con la comunidad.

4. De las respuestas obtenidas de los comandantes se puede deducir que, en su gran mayoría, ellos no alcanzan a dimensionar el impacto positivo que puede tener la presencia institucional en las casas de justicia y los centros de convivencia ciudadana. Solo algunos comandantes destinan personal suficiente para atender estos espacios de manera adecuada y han podido aprovechar esta labor para prestar un buen servicio de policía y abrir una oportunidad para transmitir una imagen positiva de la Institución.

5. En algunas casas de justicia ya se implementa el programa de mediación policial, el cual ha permitido generar una buena imagen institucional e incrementar el grado de aceptación de la Policía por parte de la comunidad. Sería conveniente extenderlo a todas las casas de justicia y los centros de convivencia ciudadana que están en funcionamiento.

6. Hasta que sea posible contar con recurso humano permanente en cada casa de justicia y centro de convivencia ciudadana, se podría pensar en una caravana móvil que acuda a desarrollar programas y proyectos que estén a su alcance; por ejemplo, una campaña de mediación o de sensibilización acerca de la violencia intrafamiliar y de prevención de la violencia ejercida contra niños, niñas y adolescentes o, en general, programas y actividades de prevención según lo que arroje el estudio de conflictividad.

7. Los estudios de conflictividad que deben elaborar las Alcaldías para diseñar sus planes, programas y presupuestos de seguridad pueden ser utilizados para 
proyectar sus estrategias tendientes a promover la seguridad y la convivencia. Así, pueden ser utilizados para el diseño de intervención policial en casas de justicia y centros de convivencia ciudadana y, por otro lado, para apoyar a los alcaldes que se decidan a tramitar su creación ante el Ministerio de Justicia y del Derecho.

8. Los miembros de la Policía Nacional que forman parte de los consejos de seguridad de sus respectivos entes territoriales podrían promover la creación o el fortalecimiento de las casas de justicia y de los centros de convivencia ciudadana ante el respectivo representante legal del municipio, distrito o departamento.

\section{Propuesta para la gestión institucional en casas de justicia y centros de convivencia ciudadana}

Como se ha expresado a lo largo de la investigación, la Policía Nacional de Colombia tiene la oportunidad de utilizar las casas de justicia y los centros de convivencia ciudadana como una estrategia para prevenir el delito y promover la convivencia ciudadana. Es importante tomar en consideración que en algunas de las casas y los centros hay servicios de conciliación y de mediación policial como un mecanismo de solución alternativa de conflictos. En ellos, los agentes de policía, después del diagnóstico del conflicto y de escuchar las versiones de las partes involucradas, proponen medios de solución con los que las partes queden satisfechas.

Otra propuesta se refiere a que la sociedad procure mecanismos naturales para la solución de los conflictos entre familiares, vecinos, amigos, empleadores y trabajadores. De allí, según Burbano, ${ }^{22}$ surgen los manuales de convivencia ampliamente utilizados en los ambientes académicos (escuelas, universidades) o en conjuntos residenciales con propiedad horizontal, para dar algunos ejemplos. Importantes iniciativas se han dado en algunos distritos como el de Medellín.

En cuanto a las colectividades, los manuales de convivencia ciudadana implican una serie de conductas y deberes cuyo cumplimiento es apreciado por los miembros de una comunidad, pues buscan generar cambios positivos de conducta que contribuyan a una mejor convivencia y constituyen uno de los instrumentos para promover

22 Milena Burbano, "La convivencia ciudadana: su análisis a partir del 'aprendizaje por reglas", Revista Colombiana de Educación, núm. 57 (2009): 33, https://www.redalyc.org/pdf/4136/413635251003.pdf (acceso agosto 15, 2019). 
la cultura ciudadana, gracias a que con ellos se puede propiciar el cumplimiento voluntario de las normas.

Antanas Mockus, durante su administración, implementó programas de pedagogía, entre ellos el denominado "apreciar las normas y admirar lo bueno". Las casas de justicia y los centros de convivencia ciudadana son espacios ideales para promover su aplicación y cumplimiento, mediante la realización de actividades pedagógicas, cursos impuestos en los comparendos, difusión de normas y atención de situaciones generadas por el incumplimiento por parte de los ciudadanos. ${ }^{23}$

El modelo unificado para la atención de casas de justicia y centros de convivencia ciudadana por parte de la Policía Nacional de Colombia implica la definición de una política con una visión nacional e incluyente respecto a las particularidades de los territorios del país. En primer lugar, es preciso definir cuáles son los programas y las actividades que deben cumplir los integrantes de esta Institución en los espacios objeto de análisis; determinar cuántos miembros de la Institución deberían prestar servicio en los mismos y los horarios en los cuales deberían estar disponibles; establecer los sistemas de información para recopilar datos de los servicios prestados con fines estadísticos y de formulación de políticas públicas en materia de seguridad y convivencia ciudadana. Así mismo, es importante determinar los perfiles y las funciones de los funcionarios que se deben destinar a atender estas instancias.

En primera instancia, los uniformados asignados a las casas de justicia y los centros de convivencia ciudadana deberán continuar atendiendo el llamado de las instituciones que pertenecen a estos espacios cuando se requiere la prestación del servicio de policía. En segundo lugar, su labor será de difusión y desarrollo de actividades que permitan la sensibilización y aplicación del Código nacional de seguridad y convivencia ciudadana, prevención del delito y orientación a poblaciones vulnerables de la comunidad.

\section{Conclusiones}

Los espacios institucionales de casas de justicia y centros de convivencia ciudadana ofrecen a la Policía Nacional de Colombia una amplia gama de oportunidades para la prestación del servicio de policía, incluso, para la promoción de la convivencia ciudadana, la prevención de la delincuencia y el fortalecimiento de la mediación

23 Burbano, "La convivencia ciudadana: su análisis a partir del 'aprendizaje por reglas", 33. 
policial, con el fin de incrementar la confianza de la población y el acercamiento de los ciudadanos con los uniformados.

La Policía Nacional de Colombia participa en las casas de justicia dando apoyo a otras entidades del orden nacional, departamental o municipal, según sea requerida. La labor que desempeña —altamente valiosa para la sociedad — debe ser sistematizada, registrada y difundida, para dar a conocer el impacto de este aporte a la sociedad, alimentar los diagnósticos de conflictividad y apoyar las decisiones de política pública en materia de seguridad y convivencia ciudadana.

En algunas casas de justicia se han instaurado los servicios de mediación policial en el marco de los métodos alternativos de solución de conflictos (MASC). Como se trata de un servicio implementado recientemente en aplicación de las disposiciones del nuevo Código Nacional de Seguridad y Convivencia Ciudadana, no aparece relacionado en el reciente Manual para la implementación y sostenibilidad del Programa nacional de casas de justicia y convivencia ciudadana, emitido por el Ministerio de Justicia y del Derecho. ${ }^{24}$ Convendría extender el servicio de mediación policial a todas las casas de justicia del país, y comenzar por aquellos lugares más apartados y con altos índices de conflictividad.

En los centros de convivencia ciudadana donde existe presencia de la Policía Nacional, se observa que su labor es respetada y que los miembros de la Institución que prestan allí sus servicios son próximos a la comunidad. Esta gestión debe ser recopilada y difundida. En relación con los servicios prestados por la Institución en estos centros, no existe un modelo de intervención ni una guía programática desde el escenario central que permita obtener resultados institucionales más claros. Si bien los programas y servicios ofrecidos obedecen a las necesidades de cada localidad, sería conveniente elaborar un programa policial nacional para intervenir en los espacios institucionales coordinados por el Ministerio de Justicia y del Derecho que nos ocupan, el cual deberá ser flexible para ajustarse a los requerimientos específicos de cada lugar.

Aunque la solicitud de crear casas de justicia o centros de convivencia ciudadana compete a la primera autoridad del municipio o distrito, el integrante de la Policía asignado a la localidad podría desempeñar una labor proactiva que impulse la articulación de las entidades del Estado del orden nacional o local y ofrecer la

24 Sánchez et al., Manual para la implementación y sostenibilidad. 
elaboración de diagnósticos de conflictividad en los cuales, según las políticas del Ministerio de Justicia y del Derecho, debe promoverse la participación ciudadana. Esta es una gran oportunidad que la ley ofrece a la Institución para alcanzar los objetivos del proceso de modernización y transformación institucional (MTI), que se viene ejecutando con proyección al año 2030.

Para tal cometido, se necesita voluntad política desde la más alta esfera, de cara al diseño y desarrollo de un plan institucional de fortalecimiento de la intervención en casas de justicia y centros de convivencia ciudadana, a partir de una reunión en la que participen los coordinadores de estas instancias, los comandantes de Policía y los representantes de las instituciones oficiales que participan en las mismas, así como miembros de las Facultades de Derecho de las universidades que tengan interés en apoyar esta gestión.

Se concluye que no se precisa un cambio de la estructura organizacional de la Institución policial, sino la implantación de un Programa nacional de apoyo a casas de justicia y centros de convivencia ciudadana, coordinado desde el escenario central, por medio de la Dirección de Seguridad Ciudadana. Así fue confirmado en entrevista al capitán Johan Álvarez, funcionario de la Oficina de Planeación de la Policía Nacional y experto en materia de estructura organizacional de la Institución, quien opinó que, con la estructura existente, se podría implementar para la Institución un Programa nacional de casas de justicia y de convivencia ciudadana, diseñado y liderado por esta Dirección. Cree que, efectivamente, estos espacios institucionales brindan una oportunidad a los miembros de la Policía Nacional para prestar a la comunidad un servicio de policía más cercano, en cumplimiento de los lineamientos del proceso de modernización y transformación institucional..$^{25}$

La implementación del Programa propuesto contribuirá en el desarrollo del Plan Estratégico de la Policía Nacional, con una "Visión 2030: policía, autoridades y comunidad", ${ }^{26}$ hacia un desarrollo sostenible, una Policía cada vez más cercana al ciudadano, más eficaz, con mayor optimización de recursos y capacidades, con el fin de generar un mayor impacto en la seguridad y convivencia de los ciudadanos. Esta perspectiva se refuerza en la misión acogida en la Resolución 00003 de 2019, por la cual se adoptan las definiciones estratégicas y el marco estratégico institucional 2019-2022:

25 Capitán Johan Álvarez, conversación personal, 6 agosto de 2019.

26 Policía Nacional de Colombia, Plan estratégico institucional comunidades seguras y en paz visión 2030 (Bogotá: Autor, 2015), 78. 
El fin primordial de la Policía Nacional es el mantenimiento de la convivencia como condición necesaria, para el ejercicio de los derechos y libertades públicas y para asegurar que los habitantes de Colombia convivan en paz fundamentada en el Código de Ética Policial. ${ }^{27}$

En conclusión, es posible afirmar que la labor de la Policía Nacional de Colombia en las casas de justicia y los centros de convivencia ciudadana donde actúa tiene un alto grado de cercanía con la ciudadanía, en atención a los servicios prestados. ${ }^{28}$

\section{Referencias}

Ahumada, Carolina y Alejandra Manzano. Diagnóstico de conflictividad. Estudio exploratorio sobre acceso a la justicia en la localidad de Sanagasta —Provincia de La Rioja. Buenos Aires: Instituto de Estudios Comparados en Ciencias Penales y Sociales, 2016.

Bello, Catalina. "Desafíos y estado futuro de la convivencia en Colombia al 2025". Criminalidad 56, núm. 2 (2014): 319-332, https://dialnet.unirioja.es/servlet/articulo?codigo=5125433 (acceso agosto 15, 2019).

Burbano, Milena. "La convivencia ciudadana: su análisis a partir del 'aprendizaje por reglas". Revista Colombiana de Educación, núm. 57 (2009): 28-45, https://www.redalyc.org/ pdf/4136/413635251003.pdf (acceso agosto 15, 2019).

Camacho Pinzón, Lina. "Acceso a la justicia en Colombia. Condiciones de posibilidad y criterios de gestión”. Tesis de maestría, Universidad Nacional de Colombia, 2013.

Colombia, Congreso de la República. Ley 136 de 1994, "Por la cual se dictan normas tendientes a modernizar la organización y el funcionamiento de los municipios". Bogotá: Diario Oficial núm. 41.377, 2 de junio de 1994.

Colombia, Presidencia de la República. Decreto 1477 de 2000, "Por el cual se adopta el Programa Nacional Casas de Justicia". Bogotá: Diario Oficial núm. 44.113, 3 de agosto de 2000.

Departamento Nacional de Planeación. Bases del Plan Nacional de Desarrollo 2018-2022. Pacto por Colombia, pacto por la equidad. Bogotá: Autor, 2018.

Díaz, Rodolfo de Jesús, Ana Rengifo, Laura Izquierdo y Liliana Buitrago. Guía para la implementación del Programa nacional de casas de justicia y centros de convivencia ciudadana. Bogotá: Ministerio de Justicia y del Derecho, 2019.

\footnotetext{
27 Ministerio de Defensa Nacional, Resolución 00003 de 2019, "Por la cual se adoptan las definiciones y el marco estratégico institucional 2019-2022 de la Policía Nacional" (Bogotá, 1 de enero de 2019), 3.

28 Capitán Álvarez, comunicación personal.
} 
Ministerio de Defensa Nacional. Resolución 00003 de 2019, "Por la cual se adoptan las definiciones y el marco estratégico institucional 2019-2022 de la Policía Nacional”. Bogotá, 1 de enero de 2019.

Páramo, Pablo y Andrea Burbano. Convivencia ciudadana en ciudades latinoamericanas. Bogotá: Universidad Pedagógica Nacional, Universidad de la República Uruguay, 2019.

República de Colombia. Constitución Política. Bogotá: Legis, 1991.

Sánchez, Jimmy, Lilián Mora, Emilsen Núñez, Tania Gómez, Laura Izquierdo, Pedro Vargas, Jhoan Tabares, Jenny Gómez y Michael Camargo. Manual para la implementación y sostenibilidad del Programa nacional de casas de justicia y convivencia ciudadana. Bogotá: Ministerio de Justicia y del Derecho, 2019. 